subgroup and meta-regression analysis across certain covariates (e.g. sex of prisoner). We hypothesise that contemporary estimates of mental disorders are higher than the general population. Background. Prevalence of mental health problems among prisoners are considerably higher than the general population; this poses an important public health concern. Individuals who require diversion to appropriate psychiatric services are becoming embroiled in the revolving door of the criminal justice system. However, there are no up-to-date reviews assessing prevalence of mental disorders across the general prison population in the UK. This study aims to address this gap.

Method. We conducted a systematic search of PsycINFO (1923 October 2019), MEDLINE (1946 - October 2019), EMBASE (1947 - October 2019) and Web of Science (all years) of articles reporting prevalence of mental disorders in UK prison populations (PROSPERO registration number: CRD42019132685). The Joanna Briggs Institute (JBI) Appraisal Checklist for Studies Reporting Prevalence Data assessed study quality and bias. Pooled prevalence of each mental disorder was calculated using Stata statistical software 16.0 via the metaprop command. Forest plots present prevalence estimates with study weights and associated 95\% confidence intervals (CI). Overall, 20 studies satisfied inclusion criteria, comprising of 12,335 prisoners across England, Wales and Scotland.

Result. We identified higher rates of neurotic disorders $(28.9 \%$, 95\% CI $0.71-74.7 \%)$, personality disorders $(23.5 \%$, 95\% CI 13.6-35.2\%), alcohol (22.7\%, 95\% CI 12.2-35.1\%) and drug dependence (26.7\%, 95\% CI $15.0-40.4 \%)$. The lowest prevalence rates included schizophrenia $(2.42 \%, 95 \%$ CI $0.78-4.84 \%)$, panic disorders $(3.88 \%$, 95\% CI 3.17\% - 4.64\%), adjustment disorders (3.83\%, 95\% CI 1.19-7.84\%) and intellectual disability (2.90\%, 95\% CI $0.90-5.80 \%)$. Meta-regressions for psychotic disorder and personality disorder revealed no significant differences across study year, sample size and gender.

Conclusion. Our prevalence estimates of mental disorders in prisons are higher than the general English population. However, we should acknowledge the influence of considerable heterogeneity. These findings demonstrate the need to quantify current prevalence of mental disorders amongst prisoners in the UK. We recommend for the government to consider performing an up-to-date census of psychiatric morbidity to facilitate service provision.

\section{A review of serious untoward incidents (SUIS) of patients with personality disorder (PD)}

Harry Reid ${ }^{1 \star}$, Tennyson Lee ${ }^{2}$, Charlotte James ${ }^{3}$, William Hancox ${ }^{4}$ and Stefanos Stoikos ${ }^{5}$

${ }^{1}$ East London NHS Foundation Trust; ${ }^{2}$ Centre for Understanding Personality Disorder (CUSP), Deancross Personality Disorder Service, ELFT NHS Foundation Trust; ${ }^{3}$ East London NHS Trust; ${ }^{4}$ North East London Foundation Trust and ${ }^{5}$ North Kensigton and Chelsea CMHT, Central and Nort West London NHS Foundation Trust, and Centre for Understanding Personality Disorder (CUSP), Deancross Personality Disorder Service, ELFT NHS Foundation Trust ${ }^{\star}$ Corresponding author.

doi: 10.1192/bjo.2021.756

Aims. The aim of this paper is to describe key findings and recommendations of SUI reports regarding patients with a diagnosis of PD in East London NHS Foundation Trust (ELFT). Patients with a diagnosis of PD are often involved in SUIs with regards to risk to themselves or others. Contributing factors might be the nature of their disorder in terms of mood instability and impulsivity, self-harming or antisocial behaviour, and the difficulties posed to assessing clinicians in predicting risk.
Background. Patients with PD present severe challenges to services. SUI findings thus serve as a lightning rod for issues in their management. With the emergence of NICE guidelines for borderline PD [2009] and antisocial PD [2009] regarding risk assessments, there has been greater optimism for management of PDs.

Method. A case series of 50 SUI reports of patients with a diagnosis of PD were identified from the governance and risk management team of ELFT. Themes were categorized as positive practice, contributory factors, and recommendations. Findings are related to guidelines in NICE and RCPsychiatry. Any patient with a diagnosis of PD (of any sub-type) that was involved in a SUI in ELFT met the inclusion criteria. There were no exclusion criteria.

Result. The most frequent themes in positive practice were 'continuity of care' and 'clinical practice'. The most frequent subthemes in clinical practice were 'assessments' and 'follow-up'. 'Continuity of care' included examples of collaborative working between various teams, as in joint assessments, good communication, and timely referrals. In contributory factors 'poor documentation' was the most frequent theme. 14 reports found no contributory factors. In recommendations the most frequent theme was the need for development and implementation of PD policies and for improved risk management.

Conclusion. NICE guidelines stress the importance of continuity of care and good clinical care and it is commendable that these were findings in positive practice. The importance of documentation being accurate and timely needs underlining in hard pressed time poor clinicians. Services would do well to review PD policies specifically regarding risk management at a wider Trust and local service level. Our findings point to the ongoing need for workforce development as recommended in the RCPSych position statement on PD published in January 2020.

\section{Obsessive-compulsive disorder: does CBT with exposure and response (ERP) prevention work?}

Jemma Reid ${ }^{1 \star}$, Naomi A Fineberg ${ }^{1}$, Lynne Drummond ${ }^{2}$, Keith Laws ${ }^{3}$, Matteo Vismara ${ }^{4}$, Benedetta Grancini ${ }^{4}$ and Davis Mpavaenda ${ }^{1}$

${ }^{1}$ Hertfordshire Partnership University NHS Foundation Trust; ${ }^{2}$ South West London and St George's Mental Health NHS Trust; ${ }^{3}$ University of Hertfordshire and ${ }^{4}$ University of Milan

${ }^{*}$ Corresponding author.

doi: 10.1192/bjo.2021.757

Aims. Since the 1970s treatment for obsessive Compulsive Disorder (OCD) has consisted of the the application of drugs acting on the serotonin system of the brain or psychological treatments using graded exposure. Although there is a large number of studies on psychological treatments, they often are underpowered. Other major methodological issues include ignoring the effects of medication during the trial, using a variety of techniques and using waiting list data as controls.

We decided to systematically review and perform a meta-analysis on randomised controlled trials (RCTs) of CBT with ERP (abbreviated to ERP)1.

Method. The study was preregistered in PROSPERO (CRD42019122311). RCTs incorporating ERP were examined. The primary outcome was the end-of-trial symptoms scores for OCD. In addition, factors which may have influenced the outcome including patient-related factors, type of control intervention, researcher allegiance and other potential forms of bias were examined. The moderating effects of patient-related and study-related factors including type of control intervention and risk of bias were also examined. 